\title{
Pi-conjugated dendrimers for solar-energy harvesting
}

Benjamin Rupert, Will Rance, Andrew Ferguson, Muhammet Kose, Sean Shaheen, and Nikos Kopidakis

A new class of materials enables a better understanding of organic photovoltaic-device physics.

Organic photovoltaics (OPV) are emerging as a low-cost technology for manufacturing solar cells on large-area flexible substrates. Potential applications include essentially all aspects of solar-energy conversion, with small-scale consumer-electronic applications as the initial goal, followed by scaling up to commercial and building applications as the technology matures. Recent developments in OPV materials and processing conditions have led to efficiencies exceeding $6 \% .{ }^{1}$ Although efficiencies as low as $7 \%$ may be sufficient for large-scale commercialization, reaching $10 \%$ would provide a much more compelling economic impetus. It has been estimated that a single-junction organic solar cell could reach $10 \%$ efficiency if the bandgap-the energy gap between the ground and excited states - of the polymer were $\sim 1.5 \mathrm{eV} .^{2}$ However, new materials are required to reach this level.

OPV devices are based on a blend of a fullerene-derivative acceptor and a conjugated (i.e., with electrons shared between a chain of continuous alternating single and double carbon bonds) polymer donor. ${ }^{3}$ Light absorption in the polymer generates a bound electron-hole pair (an exciton), that diffuses to the interface between the two materials, where it dissociates into a hole in the donor and an electron in the acceptor. The interpenetrating networks of the polymer and fullerene are responsible for conveying charges to the collecting electrodes that complete the device.

One of the main limitations of current state-of-the-art polymers for OPV is the poorly optimized overlap between their absorption spectrum and the solar spectrum. While conjugated polymers are efficient light absorbers, their optical bandgap (which determines their absorption onset) is too high to use the red and IR parts of the solar spectrum for photocurrent generation. Aiming at a guided design of donors for OPV, we recently investigated a new class of materials, conjugated den-

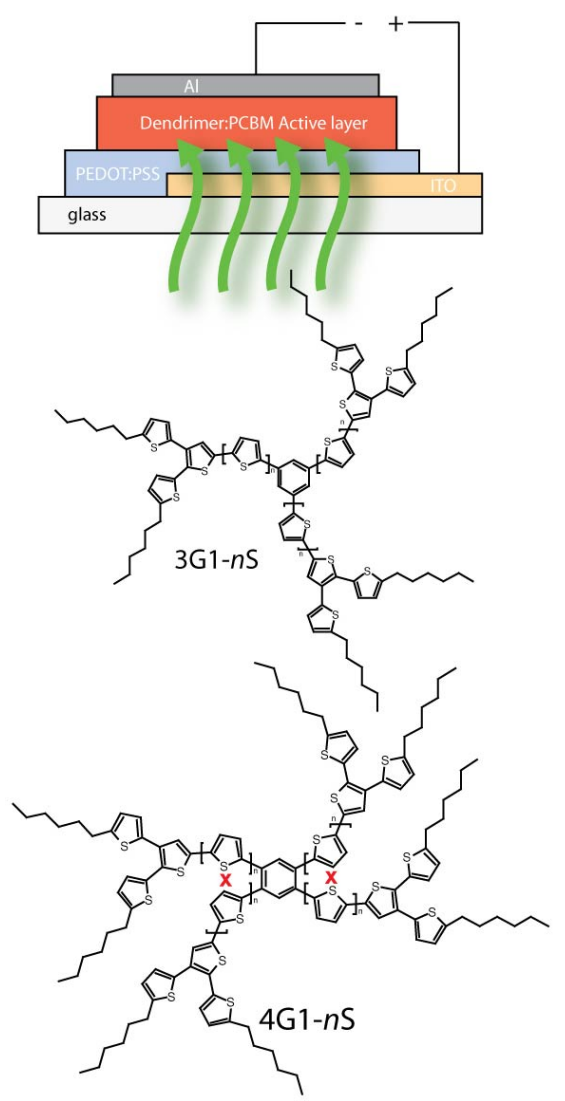

Figure 1. (Top) OPV device structure based on a dendrimer/PCBM $\left([6,6]\right.$-phenyl $C_{61}$ butyric acid methyl ester) active layer and (bottom) the molecular structures of dendrimers 3G1-nS and 4G1-nS $(n=1-3)$. The red marks on the $4 G$ dendrimer illustrate steric interactions between neighboring arms. Al: Aluminum. PEDOT:PSS: Poly $(3,4)$ thylenedioxythiophene/polystyrenesulfore acid.

drimers, with tunable absorption and electronic properties. Dendrimers (see Figure 1) are small molecules with a well-defined branched structure that allows for improved purity and batchto-batch reproducibility relative to conjugated polymers. Their

Continued on next page 
conjugated backbone makes them electronically active, and their dendritic (branched) structure allows for aliphatic end groups that enhance solubility in common organic solvents. Early, unoptimized OPV devices using phenyl-cored thiophene dendrimers and a fullerene have reached power-conversion efficiencies of $1.3 \%$ under solar irradiation. ${ }^{4}$

A desirable feature stemming from the well-defined structure of dendrimers is their amenability to computational-chemistry calculations, which we used to interpret spectroscopic data. ${ }^{5}$ We looked at the dependence of the measured optical bandgap of the dendrimers on features of the molecule such as the numbers of dendrons around the core, thiophene units between the core and the branching point, and branching points (the 'generation'). All these features have an influence on the conjugation length, which determines the effective size of the light-absorbing chromophore and the onset for optical absorption.

The notation $x \mathrm{G} y$ - $n \mathrm{~S}$ is used to name the dendrimers (see Figure 1), where $x$ is the number of arms (dendrons) around the core, $y$ the generation, and $n$ the number of thiophenes between the core and the first branching point. Note that the optical bandgap is only one of the device-relevant parameters that depend on the structure of the molecule. For example, increasing dendron length causes a decrease of the bandgap. Indeed, we found that the efficiency of OPV devices improves with increasing dendron length, ${ }^{4}$ which we attributed-at least in part-to the decreased bandgap.

Figure 2 (top) shows the measured value of the optical bandgap of the dendrimers as a function of the number of aromatic units (conjugated rings) in the longest chain. For 3G1-nS (shown in Figure 1), the dendrons are linked onto alternating carbons on the phenyl ring (they are 'metalinked') and so there is no continuous alternating single-double-bond chain along any two dendrons and the core. The longest conjugated chain is composed of any one dendron and the phenyl core, ${ }^{5}$ and the number of aromatic units in this chain is $n+3$ for 3G1-nS. For $4 \mathrm{G} 1-n \mathrm{~S}$, however, the dendrons are attached to neighboring or opposite carbons on the phenyl ring (they are 'ortho'- or 'para'-linked, respectively) and the longest conjugated chain includes two para- or ortholinked dendrons and the phenyl core. For 4G1-nS, the number of conjugated rings in the longest chain is $2(n+$ $2)+1$, which includes two dendrons and the phenyl core. In the simplest case, the number of aromatic units in the longest chain is proportional to the conjugation length that determines the bandgap and absorption onset of the molecule. Inspection of Figure 2, however, reveals that other factors also affect the conjugation length and bandgap.

As the red marks in Figure 2 (top) show, extending the dendron of the three-arm dendrimers lengthens the conjugation and
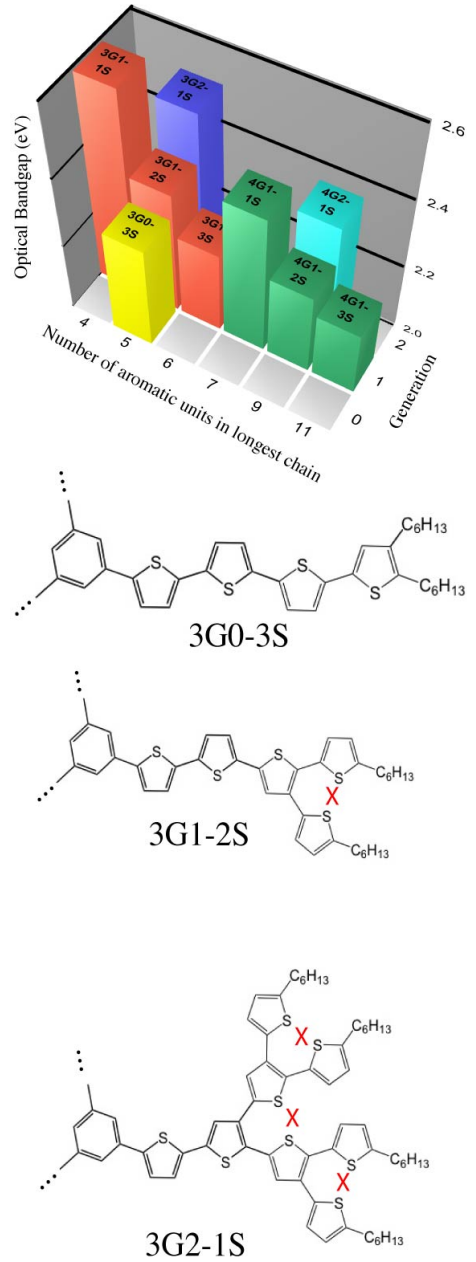

Figure 2. (Top) Optical bandgap of the dendrimers versus the basic parameters of the structure of the molecule. (Bottom) The structures of different-generation three-arm dendrimers with five aromatic units in the longest chain are also shown. The red marks indicate strong steric interactions.

reduces the band gap. Going from 3G1-3S to 4G1-1S, the number of aromatic units in the longest chain increases, because in the four-arm dendrimer the longest conjugated chain includes two dendrons and the core. However, the expected decrease of the bandgap is not observed, which means that a continuous sequence of alternating single-double bonds is not the sole determinant of the conjugation length. In this case, the presence of four arms around the core results in a structure that is congested (see Figure 1), causing the dendrons of four-arm dendrimers to twist out of the plane of the core and so break the conjugation that an ideal planar structure would have. The same principle

Continued on next page 
can be used to explain the increase of the bandgap as the generation of the dendrimer increases for a fixed number of aromatic units in the longest chain. Figure 2 shows that increasing the generation increases the number of thiophenes that are in close proximity causing steric interactions and pushing the molecule farther away from the planar structure. The latter would favor extended conjugation, causing an increase in bandgap.

We have shown how the bandgap and thus light absorption properties of OPV applications can be controlled by varying the structure of dendrimers made up of thiophene dendrons attached to a simple phenyl core. Although these still have prohibitively large bandgaps for OPV applications, substantial reductions to the bandgap have recently been achieved by substitutions of electron-withdrawing groups to the core. ${ }^{6}$ We are now undertaking fundamental spectroscopic studies to investigate the role of various excited state-species in the charge-separation process in these and other light-harvesting systems. We are also simulating exciton diffusion lengths and charge-carrier mobilities in the solid state, in conjunction with device optimization, to apply our work to pristine thin films and bulk heterojunctions.

\section{Author Information}

\section{Benjamin Rupert, Will Rance, Andrew Ferguson, Muhammet Kose and Nikos Kopidakis \\ National Renewable Energy Laboratory (NREL)}

Golden, $\mathrm{CO}$

Nikos Kopidakis is a senior research scientist. During his seven years at NREL, he has worked on dye-sensitized and organic solar cells.

\section{Sean Shaheen}

University of Denver

Denver, $\mathrm{CO}$

\section{References}

1. M. A. Green, K. Emery, Y. Hishikawa, and W. Warta, Solar cell efficiency tables (version 34), Prog. Photovolt., in press.

2. M. C. Scharber, D. Muhlbacher, M. Koppe, P. Denk, C. Waldauf, A. J. Heeger and C. J. Brabec, A new material for high-performance solar cells, Adv. Mater. 18, p. 789, 2006.

3. D. Kronholm and J. C. Hummelen, Design rules for donors in bulk-heterojunction solar cells-towards 10\% energy-conversion efficiency, SPIE Newsroom, 2008. doi:10.1117/2.1200810.1232

http:/ / spie.org/x31426.xml?highlight=x2358\&ArticleID $=x 31426$

4. N. Kopidakis, W. J. Mitchell, J. van de Lagemaat, D. S. Ginley, G. Rumbles, S. E. Shaheen, and W. L. Rance, Bulk heterojunction organic photovoltaic devices based on phenyl-cored thiophene dendrimers, Appl. Phys. Lett. 89, p. 103524, 2006.

5. M. E. Kose, W. J. Mitchell, N. Kopidakis, C. H. Chang, S. E. Shaheen, K. Kim, and G. Rumbles, Theoretical studies on conjugated phenyl-cored thiophene dendrimers for photovoltaic applications, J. Am. Chem. Soc. 129, p. 14257, 2007.

6. B. L. Rupert, W. J. Mitchell, A. J. Ferguson, M. E. Köse, W. L. Rance, G. Rumbles, D. S. Ginley, S. E. Shaheen, and N. Kopidakis, Low-bandgap thiophene dendrimers for improved light harvesting, J. Mater. Chem., in press. 